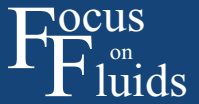

\title{
Haemodynamic stresses and the onset and progression of vascular diseases
}

\author{
JUAN C. LASHERAS
}

Departments of Mechanical and Aerospace Engineering and Bioengineering, University of California San Diego, La Jolla, CA 92093-0411, USA

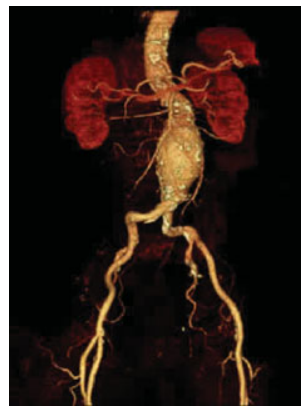

\begin{abstract}
Abdominal aortic aneurysm (AAA), a common vascular disease among the adult population, forms in the portion of the aorta below the renal arteries and upstream of its bifurcation into the two iliac arteries. While the precise cause of this vascular disease is still unknown, it is believed to be multi-factorial and predominantly degenerative, arising through a complex interplay among several biological factors as well as from specific local changes in the haemodynamic stresses on the vessels wall. Using a simple mechanical model to simulate the difference in the stiffness of the aorta and iliac arteries, Duclaux, Gallaire \& Clanet (J. Fluid Mech., 2010, this issue, vol. 664, pp. 5-32) propose a scaling argument for the transition between homogeneous and inhomogeneous deformation of an elastic tubular membrane that offers a plausible explanation for the observed localization of the AAAs. While neglecting long-term tissue remodelling and other important biological processes, the fluid mechanics model of Duclaux et al. (2010) appears to be consistent with some known associated risk factors.
\end{abstract}

Key words: biomedical flows, blood flow, flow-vessel interactions

\section{Introduction}

Blood is periodically pumped by the heart into a complex branching network of muscular elastic arteries that carry nutrients and oxygen to the tissues and organs in the body. The pulse pressure generated by the heart propagates along the network of arteries of varying size and elasticity, partially reflects at each branching point, and is damped by the time it reaches the capillaries that irrigate the tissues and organs. The blood returns to the heart through a network of veins equipped with a complex system of valves whose function is regulated by secondary muscular activity and the action of the heart itself (Nichols \& O'Rourke 1990). This active network functions as a stable feedback-control system which continuously modifies the vascular tone of the vessels in response to the short-time changes in the oxygen demand during exercise or digestion, while also regulating the long-term adaptation (remodelling) to chronic changes in haemodynamic stimuli (i.e. chronic increases in blood pressure), ageing and the onset of disease. This feedback control is mainly regulated by the vascular endothelial cells (VEC) that line the vessel's wall in a monolayer known as the vascular endothelium (intima layer). VEC sense changes in blood velocity (wall shear stresses and pressure) in a process referred to as 'mechanotransduction' (Davies 
et al. 1984), secreting prostacyclin, a vasodilator, and nitric oxide which regulate the dilatation or contraction of the arterial wall (Driss et al. 1997).

There is mounting evidence that while many of the risk factors (genetic factors, high blood pressure, high cholesterol, obesity, diabetes, cigarette smoking, high alcohol consumption, etc) and the associated biochemical mechanisms contributing to the vascular diseases act systemically throughout the entire vascular system, most cardiovascular diseases preferentially develop at specific locations in the circulatory system. This striking 'disease's localization' hints at specific haemodynamic conditions (mechanical stimuli) as one of the leading causes. For example, atherosclerosis, a process in which deposits of cholesterol, cellular waste products, calcium and other substances build up in the inner lining of a vessel causing it to harden, is preferentially localized on branches and sharp bends in the arterial system. These known areas span a total length of only several metres and represent a remarkable localization of the disease considering the approximately $100000 \mathrm{~m}$ of arteries, veins and capillaries of the human circulatory system. The disease's localization in sites characterized by unique haemodynamic conditions, occurring with high systemic concentrations of cholesterol throughout the entire circulatory system, indicates that fluid mechanical factors (haemodynamics) must play a central role (Caro, Fitzgerald \& Schroter 1971). This fact has motivated extensive studies aimed at correlating the specific haemodynamic conditions at these locations and the biochemical processes responsible for the onset and progression of the disease ( $\mathrm{Ku} 1997$; Taylor \& Draney 2004). Aneurysms (permanent dilatation of an artery) are another clear example of highly localized vascular disease which preferentially forms at specific locations of the vascular tree. Fusiform (spindle-shaped) aneurysms, such as the abdominal aortic aneurysms (AAAs), considered by Duclaux, Gallaire \& Clanet (2010), form along an extended section of an artery upstream of a bifurcation (Salsac et al. 2006; Lasheras 2007).

\section{Overview}

Aneurysms form when the arterial wall undergoes degenerative structural changes, weakens and permanently distends under the effect of the increasing wall stresses resulting from the internal blood pressure. Thus, the question is: does an aneurysm form as a result of a biological degradation process in the wall, by anomalous changes in the haemodynamic stimuli resulting from ageing, hypertension, atherosclerosis, and other risk factors which result in an unstable degenerative response on the vessel wall, by purely mechanical causes as suggested by the study of Duclaux et al. (2010) or by a combination of all of the above? The current consensus appears to be that the cause is more likely the result of a complex interplay among the degenerative biological processes triggered by inherited biochemical or structural defects, ageing, infection or disease and specific haemodynamic factors (see figure 1).

Age, hypertension and atherosclerosis are the most dominant factors in the formation of AAAs. Thus, it has been postulated that the coupling between the specific changes in the architecture of the vessel (length, diameter and wall structure) resulting from ageing and its risk factors, and the changes they induce in the haemodynamics may lead to an unstable response whereby a portion of the wall of the abdominal aorta degrades, while its diameter gradually increases (Lasheras 2007). In the portion of large elastic arteries located upstream of a bifurcation, such as the abdominal aorta, the iliac, femoral and popliteal arteries, the amplitude of the pressure wave (pulse) is considerably modified as a result of the reflection of the wave in the bifurcation 


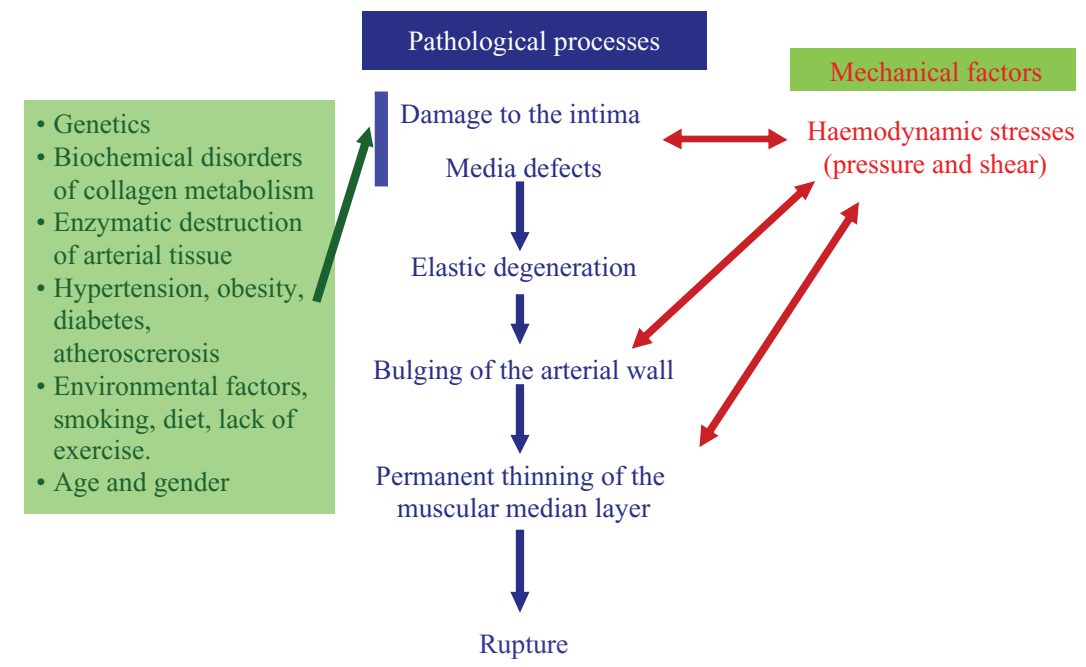

FIGURE 1. Interplay between mechanical stimuli and physiological processes.

(Fung 1993, 1997). An increase in this amplitude amplification effect may initiate and accelerate the formation of the aneurysms in the elderly population. The amplitude of the reflected wave in the abdominal aorta increases as the ratio between the cross-sectional area of the bifurcated arteries (iliacs) and the parent vessel decreases. With ageing, atherosclerosis and hypertension, these arteries also elongate slightly and gradually change their shape. Over time, the iliac arteries become more tortuous and the internal cross-section (lumen) decreases considerably. Atherosclerotic deposits of plaque in this portion of the iliacs further decrease the cross-sectional area. The progressive changes in the wave speed of the transmitted wave caused by ageing together with the increase in the ratio of cross-sectional areas may then result in an increase in peak systolic pressure in the abdominal aorta causing an increase in the wall stresses. Over time, the increased wall tension will compound and accelerate the initiation and propagation of cracks in the elastic sheets in the median layer, resulting in an irreversible decrease in the elastic recoil of the vessel and a permanent increase in the arterial diameter. This permanent diameter increase leads to a further decrease in the area ratio and thus to the unstable progression of the disease.

The response of VEC to anomalous flow shear stresses has also been suggested as a mechanism contributing to the generation of these aneurysms. During normal ageing, the abdominal aortic artery gradually undergoes conformal changes in its geometry (increasing its length and diameter, thickening of the wall, etc). Over time, the relative unconstricted nature of this artery inside the abdominal cavity may lead in some cases to the formation of bends, kinks and other morphological changes which in turn, create 'disturbed flow' conditions inside the vessel (i.e. unsteady flow separation and weak turbulence). Then, it may be argued that the anomalous response of the VEC to the high shear stresses, very low shear stresses, low, but oscillating shear stresses, and the anomalous temporal and spatial gradients of wall shear stress associated with these disturbed flow conditions could contribute to an unstable progressive degradation of the arterial wall and to the formation of the aneurysm. 


\section{Future}

As stated above, the current consensus appears to be that the cause of the formation and enlargement of AAAs is more likely the result of a complex interplay between degenerative biological processes triggered by inherited biochemical or structural defects, ageing, infection or disease and specific haemodynamic factors. Duclaux et al. (2010) provide a simple and elegant possible mechanism, but the essential issue still remains. Is the aneurysm's formation a plumbing problem? Unfortunately, for many of us fluid mechanicians in search of simple yet elegant models in biomedical applications, the answer is most likely no. The question is how far can one go with a simple mechanical model to simulate the long-term behaviour of living tissue, such as the arterial walls? Aneurysms are known to form over a period of years, while the artery is subjected to millions of cardiac cycles. The vessel walls are composed of a complex structure of living cells and a structural network of sheets and fibres of polymerized proteins actively remodelling and modifying their mechanical properties in response to changes in the mechanical stimuli from the internal blood flow. Furthermore, the arterial wall, and therefore its mechanical properties, also undergoes permanent transformations (remodelling) as a result of inflammatory processes, infection, degenerative processes and ageing.

Duclaux et al. (2010) consider an elegant yet simple and purely mechanical explanation for the enlargement of the vessel based on the mismatch in the stiffness of two sections of a tube to simulate the conditions observed in the abdominal aorta and iliac arteries. This mechanism, which retains an important element of the physics, could represent the basis of a more refined model that should include not only the response of the endothelial cells to anomalies in the flow shear stresses, but also the changes in the composition and mechanical properties of the arterial wall as it distends and remodels under increasing values of the tensional stresses.

\section{References}

Caro, C. G., Fitzgerald, J. M. \& Schroter, R. C. 1971 Atheroma and arterial wall shear observation, correlation and proposal of a shear dependent mass transfer mechanism for altherogenesis. Proc. R. Soc. Lond. Ser. B: Biol. Sci. 177 (1046), 109-159.

Davies, P. F., Dewey, C. F., Bussolari, S. R., Gordon, E. J. \& Gimbrone, M. A. 1984 Influence of hemodynamic forces on vascular endothelial function - in vitro studies of shear-stress and pinocytosis in bovine aortic-cells. J. Clin. Invest. 73 (4), 1121-1129.

Driss, A. B., Benessiano, J., Poitevin, P., Levy, B. I. \& Michel, J.-B. 1997 Arterial expansive remodeling induced by high flow rates. Am. J. Physiol. 272 (2 Part 2), H851-H858.

Duclaux, V., Gallaire, F. \& Clanet, C. 2010 A fluid mechanical view on abdominal aortic aneurysms. J. Fluid Mech. 664, 5-32.

Fung, Y. C. 1993 Biomechanics: Mechanical Properties of Living Tissues, 2nd edn. pp. xviii +568 pp. Springer-Verlag.

Fung, Y. C. 1997 Biomechanics: Circulation, 2nd edn. pp. xvii + 571 pp. Springer-Verlag.

Ku, D. N. 1997 Blood flow in arteries. Annu. Rev. Fluid Mech. 29, 399-434.

Lasheras, J. C. 2007 The biomechanics of arterial aneurysms. Annu. Rev. Fluid Mech. 39, 293-319.

Nichols, W. W. \& O'Rourke, M. F. 1990 Mcdonalds Blood Flow in Arteries: Theoretic, Experimental and Clinical Principles. Hodder Arnold Publication.

Salsac, A. V., Sparks, S. R., Chomaz, J. M. \& Lasheras, J. C. 2006 Evolution of the wall shear stresses during the progressive enlargement of symmetric abdominal aortic aneurysms. J. Fluid Mech. 560, 19-51.

TAYlor, C. A. \& Draney, M. T. 2004 Experimental and computational methods in cardiovascular fluid mechanics. Annu. Rev. Fluid Mech. 36, 197-231. 